PROCEEDINGS OF THE

AMERICAN MATHEMATICAL SOCIETY

Volume 132, Number 7, Pages 2121-2125

S 0002-9939(04)07340-X

Article electronically published on February 6, 2004

\title{
ON THE NORM OF AN IDEMPOTENT SCHUR MULTIPLIER ON THE SCHATTEN CLASS
}

\author{
WILLIAM D. BANKS AND ASMA HARCHARRAS
}

(Communicated by Andreas Seeger)

\begin{abstract}
We show that if the norm of an idempotent Schur multiplier on the Schatten class $S^{p}$ lies sufficiently close to 1 , then it is necessarily equal to 1 . We also give a simple characterization of those idempotent Schur multipliers on $S^{p}$ whose norm is 1 .
\end{abstract}

\section{INTRODUCTION}

We study norms of idempotent Schur multipliers defined on the Schatten $p$-class with $1<p<\infty, p \neq 2$. For any idempotent Schur multiplier $\phi$, we show that if the norm of $\phi$ lies sufficiently close to 1 , then it is necessarily equal to 1 . More precisely, if $\phi$ is an idempotent Schur multiplier on the Schatten $p$-class, then $\phi=0,\|\phi\|=1$, or $\|\phi\| \geq 1+\eta_{p}$, where $\eta_{p}$ is a positive constant that depends only on $p$. We also obtain a simple characterization of those idempotent Schur multipliers whose norm is equal to 1 . When $p=1$ or $\infty$, these results have been obtained by Livshits [2], while for $p=2$, every nonzero idempotent Schur multiplier has norm 1 .

To state our results more explicitly, we need to fix some standard terminology. For every real number $p$ in the range $1 \leq p<\infty$, denote by $S^{p}$ the Schatten $p$-class over the Hilbert space $\ell_{2}$; it is the Banach space of all compact operators $x: \ell_{2} \rightarrow \ell_{2}$ with finite norm

$$
\|x\|_{S^{p}}=\left(\operatorname{Tr}\left(x^{*} x\right)^{p / 2}\right)^{1 / p},
$$

where $\operatorname{Tr}(\cdot)$ denotes the usual trace. For $p=\infty$, the space $S^{\infty}$ is the Banach space of all compact operators $x: \ell_{2} \rightarrow \ell_{2}$, equipped with the usual operator norm. The spaces $S^{p}, 1 \leq p \leq \infty$, were considered in [4 as noncommutative analogues for the spaces $\ell_{p}, 1 \leq p \leq \infty$ (for a more modern reference, see [3] for example).

For $1 \leq p \leq \infty$ and a positive integer $n$, let $S_{n}^{p}$ denote the Schatten $p$-class over the Hilbert space $\ell_{2}^{n}$ of dimension $n$.

In what follows, we make no distinction between an operator $x$ on $\ell_{2}$ and the corresponding matrix $\left(x_{i j}\right)_{i, j \in \mathbb{N}}$ relative to the canonical basis $\left\{e_{i j}\right\}_{i, j \in \mathbb{N}}$ of $S^{p}$.

A set-theoretic map $\phi: \mathbb{N} \times \mathbb{N} \rightarrow \mathbb{C}$ is said to be a Schur multiplier on $S^{p}$ if the associated operator $T_{\phi}: S^{p} \rightarrow S^{p}$, defined by

$$
T_{\phi}(x)=\left(\phi_{i j} x_{i j}\right)_{i, j \in \mathbb{N}}, \quad \forall x=\left(x_{i j}\right)_{i, j \in \mathbb{N}} \in S^{p},
$$

Received by the editors December 12, 2002 and, in revised form, April 21, 2003.

2000 Mathematics Subject Classification. Primary 47A30; Secondary 47B49, 47B10.

Key words and phrases. Idempotent Schur multiplier, Schatten class.

The first author was supported in part by NSF grant DMS-0070628. 
is well defined and bounded on $S^{p}$. In particular, this implies that $\phi$ itself is a bounded map. Let $\mathcal{M}\left(S^{p}\right)$ denote the space of all Schur multipliers on $S^{p}$. Then $\mathcal{M}\left(S^{p}\right)$ is a Banach algebra when it is equipped with the pointwise product and the norm

$$
\|\phi\|_{\mathcal{M}\left(S^{p}\right)}=\left\|T_{\phi}: S^{p} \rightarrow S^{p}\right\|, \quad \forall \phi \in \mathcal{M}\left(S^{p}\right) .
$$

It is well known that for pairs $1 \leq p, q \leq \infty$ with $p^{-1}+q^{-1}=1$, the algebras $\mathcal{M}\left(S^{p}\right)$ and $\mathcal{M}\left(S^{q}\right)$ can be identified isometrically. These identifications can be done via the identity map by defining the duality between $S^{p}$ and $S^{q}$ with $\langle x, y\rangle=\operatorname{Tr}\left({ }^{t} x y\right)$ for all $x \in S^{p}$ and $y \in S^{q}$.

In addition, the space $\mathcal{M}\left(S^{2}\right)$ can be identified isometrically with the Hilbert space $\ell_{2}(\mathbb{N} \times \mathbb{N})$. Consequently, when studying $\mathcal{M}\left(S^{p}\right)$ it suffices to reduce to the case where $2<p \leq \infty$.

Finally, a Schur multiplier $\phi \in \mathcal{M}\left(S^{p}\right)$ is said to be idempotent provided that $T_{\phi} \circ T_{\phi}=T_{\phi}$; clearly, this is equivalent to the condition that $\phi$ maps $\mathbb{N} \times \mathbb{N}$ into the set $\{0,1\}$. For such multipliers, one has

$$
\|\phi\|_{\mathcal{M}\left(S^{p}\right)}=\|\phi \cdot \phi\|_{\mathcal{M}\left(S^{p}\right)} \leq\|\phi\|_{\mathcal{M}\left(S^{p}\right)}^{2} .
$$

Hence, $\|\phi\|_{\mathcal{M}\left(S^{p}\right)} \geq 1$ whenever $\phi \neq 0$. Our main result is the following:

Theorem 1. For every real number $p$ with $1<p<\infty$ and $p \neq 2$, there exists a constant $\eta_{p}>0$ (depending only on $p$ ) such that for every nonzero idempotent Schur multiplier $\phi \in M\left(S^{p}\right)$ with $\|\phi\|_{\mathcal{M}\left(S^{p}\right)} \neq 1$, the following inequality holds:

$$
\|\phi\|_{\mathcal{M}\left(S^{p}\right)} \geq 1+\eta_{p} \text {. }
$$

By the remarks above, it suffices to consider the case where $2<p<\infty$, which we assume throughout the sequel.

\section{Proof of the Main Result}

The proof of Theorem 1 can be split into three pieces, as follows.

Lemma 1. Let $\Delta=\left(\Delta_{i j}\right)_{1 \leq i, j \leq 2}$ with $\Delta_{11}=\Delta_{12}=\Delta_{22}=1$ and $\Delta_{21}=0$. Then $\|\Delta\|_{\mathcal{M}\left(S_{2}^{p^{\prime}}\right)}>\|\Delta\|_{\mathcal{M}\left(S_{2}^{p}\right)}>1$ for $2<p<p^{\prime} \leq \infty$.

Proof. For every $c \in \mathbb{C}$, let $x^{(c)}=\left(x_{i j}^{(c)}\right)_{1 \leq i, j \leq 2}$, where $x_{11}^{(c)}=x_{12}^{(c)}=x_{22}^{(c)}=1$ and $x_{21}^{(c)}=c$. One has

$$
\left\|x^{(c)}\right\|_{S_{2}^{p}}=\left(\operatorname{Tr}\left(x^{(c) *} x^{(c)}\right)^{p / 2}\right)^{1 / p}=\left(\lambda_{+, c}^{p / 2}+\lambda_{-, c}^{p / 2}\right)^{1 / p},
$$

where

$$
\lambda_{ \pm, c}=\frac{1}{2}\left(3+|c|^{2} \pm \sqrt{5+8 \Re(c)+2|c|^{2}+|c|^{4}}\right) .
$$

In particular, if we choose $c=(2-p) / 2$, then

$$
\|\Delta\|_{\mathcal{M}\left(S_{2}^{p}\right)}^{p} \geq \frac{\left\|\Delta\left(x^{(c)}\right)\right\|_{S_{2}^{p}}^{p}}{\left\|x^{(c)}\right\|_{S_{2}^{p}}^{p}}=\frac{\left\|x^{(0)}\right\|_{S_{2}^{p}}^{p}}{\left\|x^{(c)}\right\|_{S_{2}^{p}}^{p}}=f(p),
$$

where $f(p)$ is the function

$$
\frac{2^{p}\left((3+\sqrt{5})^{p / 2}+(3-\sqrt{5})^{p / 2}\right)}{\left(p^{2}-4 p+16+(p-4) \sqrt{p^{2}+16}\right)^{p / 2}+\left(p^{2}-4 p+16-(p-4) \sqrt{p^{2}+16}\right)^{p / 2}} .
$$


Since $f(2)=1, f^{\prime}(2)=0$, and

$$
f^{\prime \prime}(2)=\frac{\log (3+\sqrt{5})}{3 \sqrt{5}}-\frac{\log (3-\sqrt{5})}{3 \sqrt{5}}-\frac{1}{6}>0,
$$

the Taylor expansion for $f(p)$ near $p=2$ shows that $f(p)>1$ if $2<p<2+\varepsilon$, for some $\varepsilon>0$. Thus,

$$
\|\Delta\|_{\mathcal{M}\left(S_{2}^{p}\right)}>1, \quad \forall 2<p<2+\varepsilon .
$$

Now let $p^{\prime}>p>2$ be arbitrary real numbers and let $0<\theta<1$ be chosen so that $1 / p=(1-\theta) / 2+\theta / p^{\prime}$. By the classical results of complex interpolation, we have $S_{2}^{p}=\left(S_{2}^{2}, S_{2}^{p^{\prime}}\right)_{\theta}$ isometrically (for the definition and fundamental results on complex interpolation, the reader is referred to [1]); hence it follows that

$$
\|\Delta\|_{\mathcal{M}\left(S_{2}^{p}\right)} \leq\|\Delta\|_{\mathcal{M}\left(S_{2}^{2}\right)}^{1-\theta}\|\Delta\|_{\mathcal{M}\left(S_{2}^{p^{\prime}}\right)}^{\theta}
$$

Taking $2<p<2+\varepsilon$ with $\varepsilon$ sufficiently small, and using the obvious fact that $\|\Delta\|_{\mathcal{M}\left(S_{2}^{2}\right)}=1$, the preceding relation and our results above imply that $\|\Delta\|_{\mathcal{M}\left(S_{2}^{p^{\prime}}\right)}>$ 1 for all $2<p^{\prime} \leq \infty$. Since $0<\theta<1$, the above relation further implies that $\|\Delta\|_{\mathcal{M}\left(S_{2}^{p^{\prime}}\right)}>\|\Delta\|_{\mathcal{M}\left(S_{2}^{p}\right)}$ for $2<p<p^{\prime} \leq \infty$. This completes the proof.

It has been shown in [2] that $\|\Delta\|_{\mathcal{M}\left(S_{2}^{\infty}\right)}=2 / \sqrt{3}$, which provides an upper bound for $\|\Delta\|_{\mathcal{M}\left(S_{2}^{p}\right)}$ for any $p>2$. On the other hand, in the notation of Lemma 1 and taking $c=-1$, we have for $p>2$,

$$
\|\Delta\|_{\mathcal{M}\left(S_{2}^{p}\right)} \geq \frac{\left\|x^{(0)}\right\|_{S_{2}^{p}}}{\left\|x^{(-1)}\right\|_{S_{2}^{p}}}=\left(\frac{(3+\sqrt{5})^{p / 2}+(3-\sqrt{5})^{p / 2}}{2^{p+1}}\right)^{1 / p}>\frac{\sqrt{3+\sqrt{5}}}{2^{1+1 / p}} .
$$

It remains an interesting question to determine the precise value of $\|\Delta\|_{\mathcal{M}\left(S_{2}^{p}\right)}$ for any $p$ in the range $2<p<\infty$; this will not be needed, however, in what follows.

We now define, for each $p$ in the range $2<p<\infty$,

$$
\eta_{p}=-1+\|\Delta\|_{\mathcal{M}\left(S_{2}^{p}\right)} .
$$

In view of Lemma 1, $\eta_{p}$ is strictly positive.

Definition. A map $\phi$ defined on $\mathbb{N} \times \mathbb{N}$ (or any of its subsets) is said to be trianglefree if there are no integers $i, j, k, l$ such that $\phi_{i j}=\phi_{i l}=\phi_{k j}=1$ and $\phi_{k l}=0$.

The following lemma is an easy consequence of Lemma 1 the proof is omitted.

Lemma 2. Fix $p>2$, and suppose that $\phi \in \mathcal{M}\left(S^{p}\right)$ is idempotent. If

$$
\|\phi\|_{\mathcal{M}\left(S^{p}\right)}<1+\eta_{p}
$$

then $\phi$ is triangle-free.

Finally, we have

Lemma 3. If a map $\phi: \mathbb{N} \times \mathbb{N} \rightarrow\{0,1\}$ is nonzero and triangle-free, then $\|\phi\|_{\mathcal{M}\left(S^{p}\right)}=1$ for every real number $p>2$.

Proof. For any positive integer $n$, denote by $\phi^{(n)}$ the restriction of $\phi$ to the subset $\{1,2, \ldots, n\} \times\{1,2, \ldots, n\}$ of $\mathbb{N} \times \mathbb{N}$. Recalling the well-known fact

$$
\|\phi\|_{\mathcal{M}\left(S^{p}\right)}=\sup _{n \geq 1}\left\|\phi^{(n)}\right\|_{\mathcal{M}\left(S_{n}^{p}\right)}
$$

we see that it suffices to show that $\left\|\phi^{(n)}\right\|_{\mathcal{M}\left(S_{n}^{p}\right)}=1$ whenever $\phi^{(n)} \neq 0$. 
To this end, let $n \geq 1$ be fixed with $\phi^{(n)} \neq 0$. For every integer $1 \leq i \leq n$, define the row sum

$$
c_{i}=\#\left\{1 \leq j \leq n \mid \phi_{i j}^{(n)}=1\right\} .
$$

To show $\left\|\phi^{(n)}\right\|_{\mathcal{M}\left(S_{n}^{p}\right)}=1$, we may freely permute the rows and/or the columns of $\phi^{(n)}$ in any way that we want; in particular, without loss of generality, we may assume that

$$
c_{1} \geq c_{2} \geq c_{3} \geq \ldots \geq c_{n}
$$

and that

$$
\phi_{11}^{(n)}=\phi_{12}^{(n)}=\phi_{13}^{(n)}=\ldots=\phi_{1 c_{1}}^{(n)}=1 .
$$

Since $\phi$ is triangle-free, for every $1 \leq i \leq n$ there are only two possibilities:

$(\alpha) \quad \phi_{i j}=1$ for all $1 \leq j \leq c_{1}$, and $\phi_{i j}=0$ for all $j>c_{1} ;$

( $\beta) \quad \phi_{i j}=0$ for all $1 \leq j \leq c_{1}$.

After permuting the rows if necessary, we may assume that $(\alpha)$ occurs for $1 \leq i \leq r_{1}$, and that $(\beta)$ occurs for $i>r_{1}$. Then

$$
\phi^{(n)}=\phi_{1} \oplus \phi_{1}^{\prime}
$$

where $\phi_{1}$ is an $r_{1} \times c_{1}$ rectangular matrix with every entry equal to 1 , and $\phi_{1}^{\prime}$ is an $\left(n-r_{1}\right) \times\left(n-c_{1}\right)$ rectangular matrix whose entries are equal to 0 or 1 and which is triangle-free. If $\phi_{1}^{\prime}=0$, we stop; otherwise, we repeat the same argument with $\phi^{(n)}$ replaced by $\phi_{1}^{\prime}$, obtaining

$$
\phi^{(n)}=\phi_{1} \oplus \phi_{2} \oplus \phi_{2}^{\prime} .
$$

We continue in this way until the process stops, at which point we have

$$
\phi^{(n)}=\phi_{1} \oplus \phi_{2} \oplus \ldots \oplus \phi_{s},
$$

where every $\phi_{k}, 1 \leq k \leq s$, is an $r_{k} \times c_{k}$ rectangular matrix, all of the entries of $\phi_{1}, \ldots, \phi_{s-1}$ are equal to 1 , and the entries of $\phi_{s}$ are all equal to 1 or all equal to 0 . By adding some additional zero rows and/or zero columns to $\phi^{(n)}$ if necessary, we may also assume that $r_{k}=c_{k}$ for $1 \leq k \leq s$. Then

$$
\left\|\phi^{(n)}\right\|_{\mathcal{M}\left(S_{n}^{p}\right)}=\sup _{1 \leq k \leq s}\left\|\phi_{k}\right\|_{\mathcal{M}\left(S_{r_{k}}^{p}\right)}=1
$$

and the result follows.

Theorem 1 is an immediate consequence of Lemmas 1 3 , as the reader can easily verify.

Examining the proof of Theorem 1, we see that for a nonzero idempotent Schur multiplier $\phi$, the following assertions are equivalent:

(a) for some $p>2, \phi: S^{p} \rightarrow S^{p}$ has norm 1 ;

(b) $\phi$ is triangle-free;

(c) $\phi$ is equivalent to a multiplier of the form $\phi_{1} \oplus \phi_{2} \oplus \phi_{3} \oplus \ldots$, where each $\phi_{j}$ has all of its entries equal to 1 or all of its entries equal to 0 ;

(d) $\phi: S^{\infty} \rightarrow S^{\infty}$ has norm 1;

(e) for every $p, \phi: S^{p} \rightarrow S^{p}$ has norm 1 . 


\section{ACKNOWLEDGMENTS}

We thank G. Pisier for bringing this question to our attention; the question was inspired by a talk given by V. Paulsen at the AMS Sectional Meeting held in Irvine, California, in November, 2001.

\section{REFERENCES}

[1] J. Bergh and J. Löfström, Interpolation spaces: An introduction, Grundlehren der Mathematischen Wissenschaften, Band 223, Springer-Verlag, Berlin-New York, 1976. MR 58:2349

[2] L. Livshits, A note on 0-1 Schur multipliers, Linear Algebra Appl. 222 (1995), 15-22. MR 96d:15040

[3] I. C. Gohberg and M. G. Krein, Introduction to the theory of linear nonselfadjoint operators, Translations of Mathematical Monographs, Vol. 18, American Mathematical Society, Providence, R.I., 1969. MR 39:7447

[4] R. Schatten, Norm ideals of completely continuous operators, Ergebnisse der Mathematik und ihrer Grenzgebiete, Heft 27, Springer-Verlag, Berlin-Göttingen-Heidelberg, 1960. MR 22:9878

Department of Mathematics, University of Missouri, Columbia, Missouri 65211

E-mail address: bbanks@math.missouri.edu

Department of Mathematics, University of Missouri, Columbia, Missouri 65211

E-mail address: harchars@math.missouri.edu 